www.jmscr.igmpublication.org

Impact Factor 6.379

Index Copernicus Value: 71.58

ISSN (e)-2347-176x ISSN (p) 2455-0450

crossref DOI: _https://dx.doi.org/10.18535/jmscr/v6i3.201

Journal Of Medical Science And Clinical Research

\title{
Is Enhanced Recovery after Gastric Surgery Feasible for Gastric Cancer in Our Setup
}

\author{
Authors \\ Mubashir A Shah ${ }^{1}$, Mudasir Habib ${ }^{2}$, Ishfaq A Parrah ${ }^{3}$, Sameer H Naqash \\ 1,2,3,4 Department of General and Minimally Invasive Surgery, Sher I Kashmir Institute of Medical Sciences, \\ Srinagar, Kashmir, India
}

\begin{abstract}
Background: Enhanced recovery after surgery (ERAS) is a new concept of perioperative patient care that focuses on the minimization of impact of surgery on patients homeostasis. The concept is being routinely practised in colorectal surgeries but there are only a few studies on its application and safety in Upper Gastrointestinal surgeries. We undertook this study to look for the advantages and feasibility of ERAS in Gastric Resections in our Institute.

Methods: This Prospective study consisted of 100 patients randomly assigned to two groups, ERAS group $(n=50)$ and Control Or Traditional group $(n=50)$, operated in the Department of Surgery between 2013 and 2016. All patients managed as per ERAS protocol were compared with controls in terms of time of mobilization, appearance of bowel function, initiation of enteral feeding, development of complications and length of hospital stay. Data was recorded and analysed.

Results: Patients in the ERAS group were mobile early (on zero post op day VS control on 1pod), NG tubes were removed earlier ( $2^{\text {nd }} P O D$ VS $3^{R D}-4^{T H} P O D$ ), resumed orals earlier (ON $3^{R D} P O D$ VS $\left.5^{T H} P O D\right)$, bowel functional returned back to normal much earlier than those in control group. Hospital stay was much lower in ERAS group (mean 5 days vs 8.5 days). Patients in the both groups were comparable in terms of postoperative complications .Readmission within 30 days of Discharge was higher for ERAS Group compared to Control (4\%vs0.0\%)

Conclusion: The principles of ERAS in Gastric Resections are applicable and beneficial and decreases the hospital stay without increasing the risk of complications.

Keywords: ERAS, gastrectomy, ambulation, recovery.
\end{abstract}

\section{Introduction}

Over the last 20 years, a new concept of perioperative patient care after different types of abdominal procedures has been developed and evaluated. This model of evidence based interventions referred to as Fast Track surgery, Enhanced Recovery After Surgery (ERAS) or Multimodal Rehabilitation, is mainly focused on minimization of impact of surgery on patient homeostasis $^{[1,2]}$. ERAS was first introduced by a Danish Surgeon, H Kehlet ${ }^{[3]}$, in the field of elective colorectal surgery in early $90 \mathrm{~s}$ and it rapidly gained popularity around the world. However, there have not been many studies on its safety and benefits in Upper GI Surgeries. 
The core elements of ERAS comprise preoperative, perioperative and postoperative measures. Preoperative elements include extensive preoperative counseling for sensitization to concept of ERAS, shorter preoperative fasting and preoperative oral carbohydrate loading. The perioperatve elements include thoracic Epidural analgesia and minimal invasive surgical techniques. The postoperative elements include early ambulation, avoiding opiod analgesia, avoiding or early removal of nasogastric or nasojejunal tubes and abdominal drains and early initiation of oral feedings.

In Kashmir which is the northernmost state of India, Stomach cancer is the most commonly reported cancer amongst males $(25.2 \%)$ and the third most common cancer in females $10.4 \%{ }^{[4]}$. With only one Government hospital providing specialized care for these cancer patients, the burden of disease is overwhelming where the patients suffer from long waiting periods. Thus, the application of the concept of ERAS may be even more beneficial in our setup if it is proven to be safe and feasible.

\section{Methods}

Between August2013 and May 2016, we conducted a randomized prospective study on gastric cancer patients by randomly allocating them, by systematic randomized sampling, into two groups- ERAS Group and Traditional group. Inclusion criteria required that patients (1) had a documented diagnosis of gastric adenocarcinoma, (2) were scheduled to undergo open radical gastric surgery. Patients who underwent emergency surgery for outlet obstruction, bleeding or perforation were excluded. Mentally incompetent, pregnant, younger than 20 years and those patients that were found unresectable at exploration of abdomen were also excluded.

Patients in the ERAS group were counseled in the ward in detail and the postoperative goals were predefined regarding mobilization, oral intake, analgesia and use of NG tubes and drains. Preoperative intravenous fluids were avoided and patients were allowed to take clear fluids (including Dextrose $10 \% 500 \mathrm{ml}$ if not diabetic, to load carbohydrates) upto 2to 3 hours prior to surgery. Appropriate antibiotic prophylaxis was given. Intraoperatively, these patients received epidural analgesia through catheter unless contraindicated; were given local anaesthesia infiltration (xylocaine 2\%) at the wound site, were operated through upper midline incision. Standard radical gastrectomies were performed with curative intent. Ample measures were taken to avoid hypothermia intraoperatively. Abdominal drains, nasogastric tubes and catheters were used. Postoperatively narcotic analgesics were avoided. Patients were encouraged to sit on the bed in the evening and mobilized out of bed on the $1^{\text {st }}$ post operative morning. Thromboprophylaxis was started from $1^{\text {st }}$ POD and continued for 5 days. Urinary catheters were removed on $1^{\text {st }}$ POD and epidural catheters on $2^{\text {nd }}$ POD. Nasogastric or nasojejunal tubes were removed within 2 days in most of the patients. Patients were encouraged to take clear liquids sips by the evening of $2^{\text {nd }}$ POD and light orals on $3^{\text {rd }}$ POD. NO predefined timing for removal of drains was set. Discharge was planned on $5^{\text {th }}$ POD depending on patients overall recovery. Postoperative complications were dealt accordingly. Discharge criteria included (1) good pain control with oral analgesic, (2) taking solid food and no IV fluids, (3) independently mobile or as prior to admission, and (4) all of the above and willing to go home.

Patients in the control group were managed as per the existing protocol in our hospital. These patients were kept fasting overnight prior to the day of surgery. None of the patients received epidural analgesia. Intra operatively and Postoperatively all patients received narcotic analgesia. Urinary catheters, NG tubes and abdominal drains were used liberally .No set timeline or criteria was used in their removal. Most of the patients were put on parenteral nutrition till the oral nutrition was started. No set criteria for discharge of these patients existed and 
discharge was primarily based on consultants review.

All the patients were followed in OPD and any readmission within 30 days was noted and reasons ascertained and treated.

Patients in the study group were carefully matched with controls.

The results were compiled and analyzed statistically. Data was described as mean+- $\mathrm{Sd} / \mathrm{SE}$ and percentages. The intergroup variance was measured by Students t-test and Fishers Exact test, Mann- whitney $U$ test and $p$ - value of $<0.05$ was considered significant.

\section{Results}

The study included a total of 100 patients with 50 patients in ERAS group and 50 in control group.

The two groups were similar in age distribution with mean age of 53.9 10.08years in ERAS and 52.79 .8 years in control group. 35 patients $(70 \%)$ in ERAS and 39 (78\%)in control group were males. Hypertension was the most common associated comorbidity present in 56\% ERAS and $46 \%$ control group.

Preoperative Carbohydrate loading was possible in $86 \%$ of ERAS patients, diabetic patients (14\%) were excluded.NPO duration was $6 \mathrm{hrs}$ or less in ERAS group whereas it was around 12 hours in control.

Distal subtotal Gastrectomy was done in 25 patients (50\%) in ERAS and $28(56 \%)$ in control group. Total Gastrectomy was done in 18 (36\%) in ERAS and 14(28\%) in control and Proximal Gastrectomy was done in $7(14 \%)$ and $8(16 \%)$ respectively.

On the $1^{\text {st }}$ POD $88 \%$ of ERAS patients were ambulatory whileas only $36 \%$ of control group were ambulatory on 1 st POD which was a significant difference.

The Nasogastric (NG) ornasojejunal (NJ) tubes were removed in 45 (90\%) ERAS group patients by $2^{\text {nd }}$ POD. Rest had their tubes removed by $4^{\text {th }}$ day. On the other hand, only 20(40\%) patients had their tubes removed by 4 th day and all the tubes were removed by $9^{\text {th }}$ day. This was a statistically significant difference ( $\mathrm{p}$ value $<0.001$ ) in terms of duration of $\mathrm{NG} / \mathrm{NJ}$ tube placement.

Table: 1 Demographic and clinical parameters of two groups

\begin{tabular}{|c|c|c|}
\hline & $\begin{array}{l}\text { ERAS } \\
\text { Group }\end{array}$ & $\begin{array}{l}\text { Traditional } \\
\text { Group }\end{array}$ \\
\hline Mean Age in years & 53.9 & 52.7 \\
\hline Sex- & & \\
\hline Males & $35(70 \%)$ & $39(78 \%)$ \\
\hline Females & $15(30 \%)$ & $11(22 \%)$ \\
\hline \multicolumn{3}{|l|}{ Comorbidity- } \\
\hline Nil & $9(18 \%)$ & $8(16 \%)$ \\
\hline Hypertention & $28(56 \%)$ & $23(46 \%)$ \\
\hline Diabeties Mellitus & $7(14 \%)$ & $11(22 \%)$ \\
\hline Cardio Respiratory & $5(10 \%)$ & $7(14 \%)$ \\
\hline Hypothyroidism & $6(12 \%)$ & $5(10 \%)$ \\
\hline \multicolumn{3}{|l|}{ ASA Grading- } \\
\hline I & $15(30 \%)$ & $13(26 \%)$ \\
\hline II & $33(66 \%)$ & $35(70 \%)$ \\
\hline III & $2(4 \%)$ & $2(4 \%)$ \\
\hline \multicolumn{3}{|l|}{ Location oftumor- } \\
\hline Antropyloric & $28(56 \%)$ & $32(64 \%)$ \\
\hline Body And Fundus & $9(18 \%)$ & $9(18 \%)$ \\
\hline Incisura & $8(16 \%)$ & $6(12 \%)$ \\
\hline Ge Junction & $3(6 \%)$ & $2(4 \%)$ \\
\hline Diffuse & $2(4 \%)$ & $1(2 \%)$ \\
\hline \multicolumn{3}{|l|}{ Stage of tumour- } \\
\hline I & $0(0 \%)$ & $0(0 \%)$ \\
\hline II & $10(20 \%)$ & $8(16 \%)$ \\
\hline III & $40(80 \%)$ & $42(84 \%)$ \\
\hline \multirow{4}{*}{$\begin{array}{l}\text { Type of surgery done- } \\
\text { Distal Subtotal - } \\
\text { Gastrectomy } \\
\text { Total Gastrectomy } \\
\text { Upper Partial Gastrectomy }\end{array}$} & & \\
\hline & $25(50 \%)$ & $28(56 \%)$ \\
\hline & & \\
\hline & $\begin{array}{c}18(36 \%) \\
7(14 \%)\end{array}$ & $\begin{array}{c}14(28 \%) \\
8(16 \%)\end{array}$ \\
\hline
\end{tabular}

Urinary indwelling catheter was removed in all patient in ERAS on $1^{\text {st }}$ POD . $40(80 \%)$ patients in control group were of urinary catheter.

Abdominal drains were removed on $4^{\text {th }}$ day in $88 \%$ in ERAS group whileas it was removed in only $18 \%$ in control.

Oral feeds were started in 42 patients in ERAS on $3^{\text {rd }}, 6$ patients on $4^{\text {th }}$ and 2 on $5^{\text {th }}$ POD. In control group oral feeds were resumed on $6^{\text {th }}$ POD in 30 patients and in remaining 20 patients by $9^{\text {th }}$ POD. In ERAS group all the patients passed flatus by $4^{\text {th }}$ POD whileas all the patients passed flatus by $5^{\text {th }}$ POD . 
Table: 2 Post Operative Events and Course

\begin{tabular}{|c|c|c|}
\hline & ERAS Group & $\begin{array}{c}\text { Traditional } \\
\text { Group }\end{array}$ \\
\hline $\begin{array}{c}\text { Ambulation Initiated- } \\
\text { POD } 1 \\
\text { POD } 2\end{array}$ & $\begin{array}{c}44(88 \%) \\
6(12 \%)\end{array}$ & $\begin{array}{l}18(36) \\
32(64)\end{array}$ \\
\hline $\begin{array}{c}\text { Ryles Tube Removed- } \\
\text { POD } 2 \\
\text { POD } 2 \\
\text { POD } 3 \\
\end{array}$ & $\begin{array}{c}45(90 \%) \\
3(6 \%) \\
2(4 \%) \\
\end{array}$ & $\begin{array}{c}0(0) \\
20(40) \\
30(60) \\
\end{array}$ \\
\hline $\begin{array}{l}\text { PUC Removed- } \\
\text { POD } 1 \\
\text { POD } 2\end{array}$ & $\begin{array}{c}50(100 \%) \\
0(0 \%)\end{array}$ & $\begin{array}{l}40(80 \%) \\
10(20 \%)\end{array}$ \\
\hline $\begin{array}{c}\text { Drain Removed- } \\
\text { POD } 4 \\
\text { POD } 5 \\
\text { POD } 50 r>\end{array}$ & $\begin{array}{c}44(88 \%) \\
6(12 \%) \\
0(0 \%) \\
\end{array}$ & $\begin{array}{c}9(18 \%) \\
20(40 \%) \\
21(42 \%)\end{array}$ \\
\hline $\begin{array}{c}\text { Oral Feeding Started - } \\
\text { POD } 3 \\
\text { POD } 4 \\
\text { POD } 5 \text { or }> \\
\end{array}$ & $\begin{array}{c}42(84 \%) \\
6(12 \%) \\
2(4 \%) \\
\end{array}$ & $\begin{array}{c}0(0) \\
20(40) \\
30(60) \\
\end{array}$ \\
\hline $\begin{array}{c}\text { First Flatus Pased } \\
\text { POD } 03 \\
\text { POD } 4 \\
\text { POD } 5 \text { or }> \\
\end{array}$ & $\begin{array}{c}25(50 \%) \\
25(50 \%) \\
0(0 \%) \\
\end{array}$ & $\begin{array}{c}0(0 \%) \\
35(70 \%) \\
15(30 \%) \\
\end{array}$ \\
\hline Mean Hospital Stay & 6.42 days & 9.66 day \\
\hline
\end{tabular}

In Control group, 2 had wound infection, 2 had bowel obstruction and one duodenal blow out, 2 had respiratory complications and one patient suffered DVT. In ERAS group5 patients had complications, 2 had wound infections and one had persistent vomiting and one had duodenal stump leak, 1 had respiratory complication. None of the patients in either group needed reexploration. The difference between the two groups in terms of complications was not statistically significant.

There was statistically significant difference between ERAS and Control group in terms of postoperative hospital stay. In the ERAS group the mean hospital stay was 5 days +-1 while as it was 8 days +-2 days

Table: 3 Post Operative Complications in two Groups

\begin{tabular}{|c|c|c|}
\hline & $\begin{array}{c}\text { ERAS } \\
\text { Group }\end{array}$ & $\begin{array}{c}\text { Traditional } \\
\text { Group }\end{array}$ \\
\hline Surgical- & & \\
Wound Infection & $2(4 \%)$ & $2(4 \%)$ \\
Bowel Obstruction & $1(2 \%)$ & $2(4 \%)$ \\
Anastomotic Leak & $1(2 \%)$ & $1(2 \%)$ \\
\hline General Complications- & & \\
Respiratory & $1(2 \%)$ & $2(4 \%)$ \\
Dvt & $0(0 \%)$ & $1(2 \%)$ \\
\hline Perioperative- & & \\
Mortality & $0(0 \%)$ & $0(0 \%)$ \\
\hline
\end{tabular}

\section{Discussion}

Evidence is accumulating in literature that significant improvement in outcome of surgical procedures can be achieved by implementing multimodal perioperative care protocols. These fast track or enhanced recovery programmes are composed of a number of elements, each of which is supported by scientific evidence. The main rationale for implementing enhanced recovery programs in surgical practice is that an improved and shorter recovery period would reduce a patient's needs to stay in hospital and thus reduce the length of hospitalization-a simple concept, but particularly revolutionary as it refers exclusively to a reduction in length of stay by influencing the number of the high intensity first days after surgery.

ERAS has been widely accepted as effective perioperative management protocol in colorectal surgeries. However, its application in Upper Gastrointestinal surgeries is still not widespread. The literature, though limited, clearly establishes it role in Upper GI surgeries too.

Sherikashmir Institute of Medical Sciences is the only tertiary care hospital and a regional cancer center in Kashmir, the Northermost state of India, that caters to the malignancy patients. Having the highest incidence of cancer stomach in India, there is a long waiting period before the patients are taken up for treatment. ERAS is one of the measures to increase the turnover of such patients in an effective and safe manner.

This study was conducted over three years on one hundred patients that were operated for gastric cancer. Patients were randomly allocated by systemic randomization sampling method into two groups- ERAS or Study group and Traditional or control group with 50 patients in each group. The two groups were similar in age distribution with mean age of 53.9 10.08years in ERAS and 52.7 9.8 years in control group. In terms of mean age, our study was comparable to a study conducted by JING Xiang Song et al on ERAS. 35 patients $(70 \%)$ in ERAS and 39 (78\%)in control group were males. 
Patients in both the groups underwent EGD and were investigated properly to arrive at a final diagnosis. There was no significant statistical difference between the groups in terms of EGD findings and investigations. There is no detailed data available in the literature on ERAS so as to make a comparision.

In our study $66 \%$ of patients were ASA-II, 30\% were ASA-I and $4 \%$ were ASA-III in ERAS group. In Traditional group $70 \%$ were ASA-II, $26 \%$ were ASA-I and $4 \%$ were ASA-III. None of the either group were more than ASA-III. Both the groups were comparable in terms of ASA gradins with no significant statistical difference between the two groups. In the regard our study was comparable to study conducted by jing-xiangsong et $\mathrm{al}^{[5]}$.

The post operative elements of FAST TRACK protocol like early ambulation, removal of ryles tube, urinary catheter, drains, intitiation of early orals, passing of first flatus and early discharge from hospital was comparable various studies on $\operatorname{ERAS}^{[6,7,8]}$.

We did not find any significant difference between the two groups with respect to surgical compilations like wound infection, obstruction and anastomotic leak, in this respect our study was comparable to other studies on ERAS ${ }^{[9,10,11]}$. We found slightly increased incidence of respiratory complications in traditional group and also one patient suffered DVT in traditional group. The less incidence of these complications in ERAS group can be explained by using epidural analgesia, early ambulation, early removal of ryles tube and drains in ERAS group.

\section{Conclusion}

From this study we concluded that ERAS leads to a faster recovery, early return of gut function, less respiratory complications, shorter hospital stay and decreased overall cost of treatment .we found the principles of ERAS are applicable in our set up and will be most beneficial in view of continuously growing pressure as a result of increasing number of gastric cancer patients..

\section{References}

1. Kehlet H: Fast-track Surgery- an update on physiological care principles to enhance recovery. Langenbecks Arch Surg 2011; 396:585-590.

2. Olsen MF, Wennberg E: Fast-track concepts in major open upper abdominal and thoracoabdominal.

3. Kehlet H, Wilmore DW: Evidence - based Surgical care and the evolution of fasttrack Surgery. Am Surg 2008;248:189198.

4. M.A. Qurieshi, M.A .Masoodi, S.A.Kadla, S.Z. Ahmad, and P. Gangadharan, “ Gastric cancer in kashmir”, Asian Pacific JOURNAL OF Cancer prevention, vol. 12, no. 1, pp. 303-307, 2011.

5. Jing-xiang Song, Xiao-huangTu, Bing Wang, Chen Lin, Zai-zhong Zhang, LiYing L and Lie Wang "Fast track" rehabilitation after gastric cancer resection: experience with 80 consecutive cases. BMC Gastroenterology 2014,14:147

6. Kim JW, Kim WS, Cheong JH, Hyung WJ, Choi SH, et al.(2012) safety and efficacy of fast-track surgery in laparoscopic distal gastrectomy for gastric cancer: a randomized clinical trail. World J Surg 36:2879-2887.

7. Yang Z, Zheng Q, Wang Z(2008) Metaanalysis of the need for nasogastric or nasojejunal decompression after gastrectomy for gastric cancer. Br J Surg 95:809-816.

8. Yamada T, Hayashi T, Cho H, Yoshikawa T, Taniguchi H, et al.(2010) Usefulness of enhanced recovery after surgery protocol as compared with conventional perioperative care in gastric surgery. Gastric Cancer 15:34-41.

9. Sakakushev BE(2013) Enhanced Recovery after surgery for Gastric Cancer.JGastroint Dig Syst S 12:003

10. Suehiro T, Matsumata T, Shikada Y, Sugimachi K (2004). Accelerated 
rehabilitation with early post operative oral feeding following gastrectomy. Hepatogastroenterology 51:1852-1855.

11. Hur H, Si Y, Kang WK, Kim W, Jeon $\mathrm{HM}(2009)$ Effects of early oral feeding on surgical outcomes and recovery after curative surgery for gastric cancer: pilot study results. World J Surg 33:1454-1458. 